\title{
VARIATIONAL EQUATIONS OF LAGRANGIAN SYSTEMS AND HAMILTON'S PRINCIPLE
}

\author{
H. N. NÚÑEZ-YÉPEZ(1), JOAQUÍN DELGADO(2), AND A. L. SALAS-BRITO(3)
}

\begin{abstract}
We discuss a recently proposed variational principle for deriving the variational equations associated to any Lagrangian system. The principle gives simultaneously the Lagrange and the variational equations of the system. We define a new Lagrangian in an extended configuration space - which we call D'Alambert's - comprising both the original coordinates and the compatible "virtual displacements" joining two solutions of the original system. The variational principle is Hamilton's with the new Lagrangian. We use this formulation to obtain constants of motion in the Jacobi equations of any Lagrangian system with symmetries. These constants are related to constants in the original system and so with symmetries of the original Lagrangian. We cast our approach in an intrinsic coordinate free formulation. Our results can be of interest for reducing the dimensions of the equations that characterize perturbations in a Lagrangian control system.
\end{abstract}

\section{INTRODUCTION}

Extremum principles have a great importance in dynamics and in control [1], [2], 3. They can be used to attain general formulations of the theory and can be of assistance in the solution of particular problems [4], 泡. The purpose of this contribution is to discuss a recently proposed extremum principle which generates both the equations of motion and the so-called variational equations describing deviations between any two solutions of a Lagrangian system [6]. The variational principle is Hamilton's but with a different Lagrangian. Since we are dealing with Lagrangian dynamical systems, in which such "least action" principle is assumed to be valid, the variational equations describe deviations between extremal curves in a certain configuration space so they, slightly abusing the language, may be called the Jacobi variational equations of the Lagrangian system as we have done in [6]. Please note that though we use a notation with a strong flavour of classical mechanics 7], the formulation is not limited in any way and can be applied to non-linear evolution equations, like the Einstein, the Korteweg-DeVries, or the KamdomtsevPetviashvili equations [8]-11]. The intrinsic formulation of section 4 should make clear this matter. The variational equations can also be used to study the stability of solutions and as starting points to evaluate their Liapunov spectrum as we have succintly discussed in [6]. They are, besides, describable in the vertical extension of the Lagrangian formalism [12]. We should mention previous related work along the same lines [13] and an older contribution with different purposes 14].

Date: October 30, 2018.

1991 Mathematics Subject Classification. 49Nxx, 70H25, 70Sxx.

(1) All authors contributed equally to this paper. Partially supported by PAPIIT-IN122498.

${ }^{(2)}$ Partially supported by Conacyt grant 32167-E.

(3) Part of this work was done while visiting the Department of Physics, Emory University. 
The article is organized as follows: In section 2 we define the new Lagrangian, use it to formulate the variational principle whose extremals are solutions of the Lagrange and the variational equations of the system. In this section we also discuss basic features of the formulation. In section 3, we address the existence of constants of motion in the variational equations and its relation with constants in the original system. In section 4 we cast our approach in an intrinsic language, making connection with the symplectic manifold that can be associated with the prolongation of the original Lagrangian. If you are interested mainly in the mathematical setting of the formulation this is the section to read. In section 5 we give various examples in which the approach may be used.

\section{The VARIATIONAL PRINCIPLE}

Let us consider then a $N$-degree of freedom dynamical system endowed with a Lagrangian function $L\left(q_{a} \dot{q}_{a}, t\right), a=1 \ldots N$; defined in the tangent bundle $T Q$ of its configuration space $Q$, i.e. $L: T Q \rightarrow \mathbb{R}$. From the Lagrangian we usually construct the action 四, [5]

$$
S[q(t)]=\int_{t_{1}}^{t_{2}} L\left(q_{a} \dot{q}_{a}\right) d t
$$

where we are using square brackets to indicate that $S$ is a functional of the paths $q_{a}(t)$ joining two given points, $q_{a}\left(t_{1}\right)$ and $q_{a}\left(t_{2}\right)(a=1, \ldots, N)$, in $Q$ at two fixed instants of time $t_{1}$ and $t_{2}$. The extremalization of $S$ directly leads to the Lagrangian equations of motion of the system [equations (6) below]. Let us now consider a different configuration space $D$, comprising both the original configurations of the system plus all the possible "virtual displacements" joining any two extremal paths of (11). With the help of $L$, we can define a new (the mathematical meaning of all this should become clear in sections 4.2 and 4.3) Lagrangian $\gamma(\mathbf{q}, \dot{\mathbf{q}}, \boldsymbol{\epsilon}, \dot{\boldsymbol{\epsilon}}, t)$ [6] as

$$
\gamma(\mathbf{q}, \dot{\mathbf{q}}, \boldsymbol{\epsilon}, \dot{\boldsymbol{\epsilon}}, t) \equiv \frac{\partial L}{\partial \dot{q}_{a}} \dot{\epsilon}_{a}+\frac{\partial L}{\partial q_{a}} \epsilon_{a}
$$

here, as in all of the paper, the summation convention is implied for repeated indices. The function $\gamma: T D \rightarrow \mathbb{R}$, where $T D$ is the tangent bundle of $D$, is central in our formulation. The $2 N$-dimensional D'Alambert configuration space $D$ is assumed, mostly in sections 2 and 3 , to be coordinatized by $\left(q_{a}, \epsilon_{a}\right), a=1, \ldots, N$. The $N$ component object $\boldsymbol{\epsilon}=\left(\epsilon_{1}, \epsilon_{2}, \ldots, \epsilon_{N}\right)$ stands for the displacement from an extremal path of (1) to another, and $\dot{\boldsymbol{\epsilon}}=\left(\dot{\epsilon}_{1}, \dot{\epsilon}_{2}, \ldots, \dot{\epsilon}_{N}\right)$ for its corresponding velocity. This means that $\boldsymbol{\epsilon}$ plays the role of the variational field associated with trajectories of the original system: $\mathbf{q}^{\prime}=\mathbf{q}+\boldsymbol{\epsilon}$ and $\dot{\mathbf{q}}^{\prime}=\dot{\mathbf{q}}+\dot{\boldsymbol{\epsilon}}$, where both $\mathbf{q}$ and $\mathbf{q}^{\prime}$ are extremal paths [1], [4], [6] of the original action (1). A useful property of $\gamma(\mathbf{q}, \dot{\mathbf{q}}, \boldsymbol{\epsilon}, \dot{\boldsymbol{\epsilon}}, t)$ is that it is an explicit function of time only when $L$ is non-autonomous.

The function $\gamma$ is used to define the new ("displaced") action functional

$$
\Sigma[\mathbf{q}(t), \boldsymbol{\epsilon}(t)]=\int_{t_{1}}^{t_{2}} \gamma(\mathbf{q}, \dot{\mathbf{q}}, \boldsymbol{\epsilon}, \dot{\boldsymbol{\epsilon}}, t) d t
$$

of the paths joining two given configurations $\left(q_{1}, \epsilon_{1}\right)$ and $\left(q_{2}, \epsilon_{2}\right)$ of the varied system between two fixed instants of time $t_{1}$ and $t_{2}$. We are calling "time" the parameter appearing in (3), but it can be any other useful parameter, for example, the arc 
length, or even may be any finite set of parameters. The statement of the variational principle is just Hamilton's, that is, $\Sigma$ is extremal when the system follows its actual path in $D[6]$

$$
\delta \Sigma[\mathbf{q}(t), \boldsymbol{\epsilon}(t)]=0 .
$$

The extremalization is done varying the path but mantaining the endpoints and the time fixed. The conditions for the functional $\Sigma[q(t), \epsilon(t)]$ to be an extremum are the $2 N$ Euler-Lagrange equations 迎, [1], 114]

$$
\frac{d}{d t}\left(\frac{\partial \gamma}{\partial \dot{\epsilon}_{a}}\right)-\frac{\partial \gamma}{\partial \epsilon_{a}}=0, \quad \frac{d}{d t}\left(\frac{\partial \gamma}{\partial \dot{q}_{a}}\right)-\frac{\partial \gamma}{\partial q_{a}}=0, \quad a=1, \ldots, N
$$

or, using the definition (2) in the preceding equations, we obtain the Lagrange equations of the original system

$$
\frac{d}{d t}\left(\frac{\partial L}{\partial \dot{q}_{a}}\right)-\frac{\partial L}{\partial q_{a}}=0, \quad a=1, \ldots, N
$$

plus the linear variational equations:

$$
M_{a b} \ddot{\epsilon}_{b}+C_{a b} \dot{\epsilon}_{b}+K_{a b} \epsilon_{b}=0, \quad a=1, \ldots, N .
$$

Equations (7) describe the evolution of the deviation, $\boldsymbol{\epsilon}$, of a varied trajectory from an unperturbed one. The $N \times N$ matrices $M, C$ and $K$, are

$$
\begin{aligned}
M_{a b} & =\left(\frac{\partial^{2} L}{\partial \dot{q}_{a} \partial \dot{q}_{b}}\right), \\
C_{a b} & =\left[\frac{d}{d t}\left(\frac{\partial^{2} L}{\partial \dot{q}_{a} \partial \dot{q}_{b}}\right)+\frac{\partial^{2} L}{\partial \dot{q}_{a} \partial q_{b}}-\frac{\partial^{2} L}{\partial \dot{q}_{b} \partial q_{a}}\right], \\
K_{a b} & =\left[\frac{d}{d t}\left(\frac{\partial^{2} L}{\partial \dot{q}_{a} \partial q_{b}}\right)-\frac{\partial^{2} L}{\partial q_{a} \partial q_{b}}\right], \quad a, b=1, \ldots, N .
\end{aligned}
$$

It shoud be clear that equations (7) are the variational equations of the original system [1], [6], [8]. One of the interesting properties of these equations is that the matrices $M, C$, and $K$, and, hence, the variational equations (7), may be regarded as time-independent as long as $L$ is autonomous. This property, that does not happen in the standard description, may have important consequences in analysing perturbations to optimal control problems [3] 15] [16]. At this point, it is worthwhile to emphasize four important features:

1. The function $\gamma: T D \rightarrow \mathbb{R}$ plays the role of a new Lagrangian describing the original system plus its response to "virtual displacements" or perturbations. It therefore is also useful for studying stability [6].

2. Equations (6) and (7) are invariant under arbitrary point transformations (i.e. changes of coordinates in $Q): Q_{a}=f_{a}(q, t), a=1, \ldots, N$, where the $f_{a}$ are functions, $f_{a}: D \rightarrow D$, assumed to be invertible (i.e. $\left.\operatorname{det}(d f(q)) \neq 0, q \in D\right)$ and at least $C^{1}$. As happens in any Lagrangian description, this result is proved by the very existence of the variational principle (3); [4], 细, 17]. 
3. The $\epsilon$-derivatives of $\gamma$ reduce to corresponding $q$-derivatives of $L$

$$
\frac{\partial \gamma}{\partial \dot{\epsilon}_{a}}=\frac{\partial L}{\partial \dot{q}_{a}}, \quad \text { and } \quad \frac{\partial \gamma}{\partial \epsilon_{a}}=\frac{\partial L}{\partial q_{a}}
$$

4. As a consequence of the previous property, $\gamma$ is a first-order homogeneous function of the virtual displacements $\epsilon_{a}$ and velocities $\dot{\epsilon}_{a}$

$$
\gamma=\frac{\partial \gamma}{\partial \epsilon_{a}} \epsilon_{a}+\frac{\partial \gamma}{\partial \dot{\epsilon}_{a}} \dot{\epsilon}_{a}
$$

\section{Symmetries And CONSTANTS OF MOtion}

One of the most important consequences of any Lagrangian description using a variational principle, is the close association between the symmetries of the Lagrangian and the existence of constants of motion [18], that is, functions that are conserved along integral curves of the Lagrangian vector field. There is a controltheory version of such result, saying that every symmetry of a control system gives rise to a conservation law along biextremals [3]. This theorem makes the following results important for perturbed control systems.

The association between symmetries and conservation laws is shared by the the function $\gamma$. The precise sense is the following: Any symmetry of $L$ can be carried over to a symmetry of $\gamma$, where they imply the existence of constants of motion in the variational equations. Although a general proof can be given, we prefer to illustrate this result in what follows - though our discussion of what we have called inherited constants comes close to being an informal proof.

3.1. Invariance under time translations. If the Lagrangian of the system is autonomous, then there exist a well-known constant of motion [1], [4],

$$
H=\frac{\partial L}{\partial \dot{q}_{b}} \dot{q}_{b}-L
$$

$H$ can, in certain instances, be identified with the energy of the system.

In such autonomous case, $\gamma$ is autonomous too and the variational equations thus admit an analogous constant of motion - which can be derived in an strictly similar fashion to $H$ [1], $[4],[6]$ - namely

$$
h=\frac{\partial \gamma}{\partial \dot{q}_{b}} \dot{q}_{b}+\frac{\partial \gamma}{\partial \dot{\epsilon}_{b}} \dot{\epsilon}_{b}-\gamma
$$

Using definition (2), the constant $h$ can be recasted as [6]

$$
h=\frac{\partial \gamma}{\partial \dot{q}_{b}} \dot{q}_{b}-\frac{\partial \gamma}{\partial \epsilon_{b}} \epsilon_{b}
$$

or, using (13), as

$$
h=\frac{\partial H}{\partial \dot{q}_{b}} \dot{\epsilon}_{b}+\frac{\partial H}{\partial q_{b}} \epsilon_{b} .
$$

If we interpret $H$ as the energy, a related interpretation for $h$ is that it is the first-order energy change in going from a solution to a displaced nearby one. 
3.2. Invariance under space translations. The invariance of a system under space translations, the property frequently called by physicists the homogeneity of space, usually manifest itself in the independence of the Lagrangian on certain coordinates. When the Lagrangian does not depend on a specific coordinate $q_{s}$, the coordinate is said to be ignorable (respect to the original Lagrangian $L$ ), then its conjugate momentum is conserved. That is, if

$$
\frac{\partial L}{\partial q_{s}}=0, \quad \text { then } \quad p_{s} \equiv \frac{\partial \gamma}{\partial \dot{\epsilon}_{s}}=\frac{\partial L}{\partial \dot{q}_{s}} \quad \text { is a constant. }
$$

If $q_{s}$ is ignorable in $L$, then, as shown in equation (17), it is also ignorable in $\gamma$, and $\epsilon_{s}$ is ignorable too, so the momentum $p_{s}$ - conjugated to $\epsilon_{a}$ in $\gamma$ - is conserved (as it should be since it is conserved in the original system!). Furthermore, $q_{s}$ is also ignorable in $\gamma$, hence the quantity (the momentum, $\pi_{s}$, conjugated to $q_{s}$ in $\gamma$ )

$$
\pi_{s} \equiv \frac{\partial \gamma}{\partial \dot{q}_{s}}=\frac{\partial^{2} L}{\partial \dot{q}_{s} \partial \dot{q}_{b}} \dot{\epsilon}_{b}+\frac{\partial^{2} L}{\partial \dot{q}_{s} \partial q_{b}} \epsilon_{b}
$$

is also a constant of motion in the variational equations.

After having proved the existence of the constants $h$ (equation 14) and $\pi$ (equation 18), it is worth formulating a more general result. As we show next such theorem follows from the close relationship between solutions to equations (16) and constants of motion in the variational equations (7). We call such first integrals, inherited constants of motion.

3.3. Inherited constants of motion. Let us consider any constant of motion, $J(\mathbf{q}, \dot{\mathbf{q}})$, of the original set of equations (6). If we evaluate it on two nearby solutions of (6), $\mathbf{q}$ and $\mathbf{q}^{\prime}=\mathbf{q}+\boldsymbol{\epsilon}$, separated by the Jacobi field $\boldsymbol{\epsilon}$, the difference, $j(\boldsymbol{\epsilon}, \dot{\boldsymbol{\epsilon}}) \equiv$ $J\left(\mathbf{q}^{\prime}, \dot{\mathbf{q}}^{\prime}\right)-J(\mathbf{q}, \dot{\mathbf{q}})$, between these constant quantities is also trivially a constant,

$$
\frac{d j(\boldsymbol{\epsilon}, \dot{\boldsymbol{\epsilon}})}{d t}=\frac{d}{d t}\left[J\left(\mathbf{q}^{\prime}, \dot{\mathbf{q}}^{\prime}\right)-J(\mathbf{q}, \dot{\mathbf{q}})\right]=0 .
$$

The constant, $j[\boldsymbol{\epsilon}]$, can be expressed as

$$
j[\boldsymbol{\epsilon}]=\left(\frac{\partial J}{\partial q_{a}} \epsilon_{a}+\frac{\partial J}{\partial \dot{q}_{a}} \dot{\epsilon}_{a}\right) .
$$

This is precisely the form of $h$ in equation (16).

Equation (20) tells us how, given both a solution of equations (5) and any one of its constants of motion, we can obtain a constant of motion in equations (7). Equation (20) can be directly proved to be a constant by computing its time derivative. This result establishes a direct relationship of constants in the variational equations, like $j$, to constants in the original Lagrangian. Related results are discussed in $[7], 19]$.

Furthermore, as in some non-linear evolution equations the constants of motion, $J[q(t)]$, are functionals (and not functions) of the solutions of (6), we need to pinpoint that the constant $j[\epsilon(t)]$ becomes a functional of the Jacobi fields $\epsilon(t)$, which must be given thus by

$$
j[\epsilon(t)]=\int \frac{\delta J[q(t)]}{\delta q(\tau)} \epsilon(\tau) d \tau
$$


where $\delta J[q(t)] / \delta q(\tau)$ stands for the functional derivative of the constant functional $J[q(t)][20]$ [21].

Having stablished the existence of the inherited constants (20) [or (21)], it should be clear that any symmetry of the original Lagrangian is reflected in the existence of another constant in the Jacobi variational equations. Thus, Noether's theorem also holds for the variational equations and can be used to reduce the dimensions of the variational system [6]; [3]. Related results are discussed in [7, section 111]; 11 .

\section{INTRINSIC FORMULATION}

In this section we formulate in an intrinsic manner our variational approach. First we consider an arbitrary vector field $Y$ (at least $C^{1}$ ) defined on a manifold $M$ and recall the constructions of the variational vector field $T(Y)$ defined on the tangent bundle $T M$ and the adjoint variational vector field $T^{*}(Y)$ defined on the cotangent bundle $T^{*} M$. As it is well known [2], 15], 16, 22], 23], $T^{*}(Y X)$ posseses a natural structure: it is a Hamiltonian vector field with respect to the the canonical symplectic form on $T^{*} M$. On the other hand the variational vector field $T(Y)$ does not have a natural Lagrangian structure due to the fact that the Hamiltonian for $T^{*}(Y)$ is linear in the momenta and so there is no natural Legendre transform $T M \rightarrow T^{*} M$ defined.

Our main interest is in the case of $M=T Q$, i.e. the tangent bundle of a configuration space, and $Y=Y_{L}$, a Lagrangian vector field on $T Q$ for the function $L: T Q \rightarrow \mathbb{R}$. We recall that if $L$ is not degenerate, the pull back of the canonical symplectic form $\omega_{0}$ in $T^{*} Q$ under the Legendre transformation $\mathcal{L}(L): T Q \rightarrow T^{*} Q$, $\omega_{L}=\mathcal{L}^{*} \omega_{0}$, makes $\left(T Q, \omega_{L}\right)$ into a symplectic manifold and the Lagrangian vector field $Y_{L}$ is Hamiltonian for the energy function $H: T Q \rightarrow \mathbb{R}$. Also $Y_{L}$ is a second order differential equation: $\tau_{Q_{*}} Y_{L}=Y_{L} \circ \tau$ where $\tau_{Q}: T Q \rightarrow Q$ is the projection.

From what was said in the first paragraph of this section, the adjoint variational vector field $T^{*}\left(Y_{L}\right)$, defined on $T^{*}(T Q)$, has a natural Hamiltonian form. We want to dilucidate what the structure of the variational vector field $T\left(Y_{L}\right)$, defined on $T(T Q)$, is. A naive guess is that $T\left(Y_{L}\right)$ should be Lagrangian and the Lagrangian should be the prolongation [15], 22] $\dot{L}: T(T Q) \rightarrow \mathbb{R}$ of $L: T Q \rightarrow \mathbb{R}:$ $\dot{L}(\xi)=d L_{\pi(\xi)}(\xi)$, for $\xi \in T(T Q)$. A moment of caveat shows that this cannot be true. In fact, taking coordinates $(q, \dot{q}, \epsilon, \dot{\epsilon})$ for $T(T Q)$, and $(q, \dot{q}, p, \pi)$ in $T^{*}(T Q)$, the prolongation is given by

$$
\frac{d L}{d t}(q, \dot{q}, \epsilon, \dot{\epsilon})=\frac{\partial L}{\partial q}(q, \dot{q}) \epsilon+\frac{\partial L}{\partial \dot{q}}(q, \dot{q}) \dot{\epsilon}
$$

and its Legendre transform $T(T Q) \stackrel{\mathcal{L}(\dot{L})}{\longrightarrow} T^{*}(T Q)$, is given by

$$
\begin{aligned}
& p=\frac{\partial \dot{L}}{\partial \epsilon}=\frac{\partial L}{\partial q}, \\
& \pi=\frac{\partial \dot{L}}{\partial \dot{\epsilon}}=\frac{\partial L}{\partial \dot{q}}
\end{aligned}
$$

but then the pull back of $\theta_{0}=p d q+\pi d \dot{q}$ is 


$$
\theta_{\dot{L}}=\mathcal{L}(\dot{L})^{*} \theta_{0}=(p \circ \dot{L}) d q+(\pi \circ \dot{L}) d \dot{q}=\frac{\partial L}{\partial q} d q+\frac{\partial L}{\partial \dot{q}} d \dot{q}
$$

that is $\theta_{\dot{L}}=d L \circ \tau_{T Q}$, there $\tau_{T Q}: T(T Q) \rightarrow T Q$ is the projection. This in turn implies $d \theta_{\dot{L}} \equiv 0(!)$. Thence the interpretation is untenable.

4.1. The variational and adjoint variational vector fields. For setting straight the formalism, let us recall briefly the appropriate construction: Let $M$ be a manifold and let $\tau_{M}: T M \rightarrow M, \tau_{M}^{*}: T^{*} M \rightarrow M$ be the tangent and cotangent bundles, respectively. Given a vector field on $M$, that is a section $Y: M \rightarrow T M$, the variational vector field associated to $Y$, herein denoted by $T(Y)$, is defined as follows: Given $m \in M$ there exists a neighborhood $B$ of $m$ and $\epsilon>0$ such that the local flow $\Phi_{t}: B_{\delta}(m) \rightarrow M$ is defined for $t \in(-\epsilon, \epsilon)$. The lift $T\left(\Phi_{t}\right)$ : $\left.T M\right|_{\pi^{-1}(B)}: \pi^{-1}(B) \rightarrow T M$ then defines a local flow and its infinitesimal generator is the variational vector field $T(Y)$. Similarly, the lifting to the cotangent bundle $T^{*}\left(\phi_{t}\right):\left.T M\right|_{p^{-1}(B)}: \pi^{-1}(B) \rightarrow T^{*} M$ defines a local flow and its infinitesimal generator is called the adjoint variational vector field $T^{*}(Y)$.

The names are well suited since in local coordinates, $(x, v)$ for $T M$ and $(x, p)$ in $T^{*} M$ (we think $x, v$ as column vectors; $p$ a row vector), the variational and adjoint variational vector fields are given respectively as

$$
\begin{array}{lll}
\dot{x}=f(x), & & \dot{x}=f(x), \\
\dot{v}=D f(x) v ; & (26 . a) & \dot{p}=-p D f(x)
\end{array}
$$

Actually the adjoint variational vector field (26.b) admits the Hamiltonian form

$$
\begin{aligned}
& \dot{x}=\frac{\partial H}{\partial x}, \\
& \dot{p}=-\frac{\partial H}{\partial x} .
\end{aligned}
$$

where

$$
H(x, p)=p f(x) .
$$

This form is compatible with the standard symplectic form on $T^{*} M$. As we mentioned earlier in this section, there is no natural Lagrangian structure for (26. a) since the Legendre transformation $(x, p) \stackrel{\mathcal{L}^{-1}(H)}{\longrightarrow}(x, v)$ is not defined due to the linear dependence of $H$ on the momenta (29).

4.2. Prolongation of a system. There is a related concept of extending a given system known as its prolongation [3], [15], [16]. The motivation comes from the following argument: if the system is given by $\dot{x}=f(x)$, then by taking time derivatives along solutions one gets

$\ddot{x}=D f(x) \dot{x}=D f(x) f(x), \dddot{x}=D^{2} f(x) \cdot(D f(x), f(x)+D f(x) \cdot(D f(x) \cdot f(x))$, etc. 
Considering the first prolongation $\ddot{x}=D f(x) f(x)$, it can be written as the first order system

$$
\begin{aligned}
\dot{x} & =v, \\
\dot{v} & =D f(x) \cdot f(x) .
\end{aligned}
$$

A natural question is if this system admits a Lagrangian structure.

Proposition 1. Consider the system $\dot{x}=f(x)$ and suppose that $\frac{\partial f_{i}}{\partial x_{j}}=\frac{\partial f_{j}}{\partial x_{i}}$, that is $D f(x)$ be symmetric. Then the first prolongation of the system (31) is Lagrangian for the function

$$
L(x, v)=\frac{1}{2}|v|^{2}+\frac{1}{2}|f(x)| .
$$

The proof is elementary and will be omited.

The symmetry condition can be rephrased in terms of the graph

$$
G_{f}=\left\{\left(x, f(x)^{T}\right) \mid x \in \operatorname{Dom} f\right\} \subset R^{n} \times\left(R^{n}\right)^{*}
$$

as being Lagrangian [24], [15] (here the notation $f(x)^{T}$ means simply that $f(x)$ is viewed as a covector).

If $f: M \rightarrow N$, its prolongation [3] [15] is the map $\hat{f}: T(M) \rightarrow T^{*}(N)$ defined as follows: For $\xi \in T_{x} M$ and $\alpha \in T_{f(x)}^{*}(M)$, let $\langle$,$\rangle denote the natural pairing, then$

$$
\hat{f}(\xi)(\alpha)=\left\langle f_{* x}(\xi), \alpha\right\rangle .
$$

In local coordinates $(x, v) \in \mathbb{R}^{n} \times \mathbb{R}^{n}$ for $T M$ and $(y, p) \in \mathbb{R}^{n} \times\left(\mathbb{R}^{n}\right)^{*}$ for $T^{*}(N)$ the first prolongation is given by

$$
\begin{aligned}
& y=f(x), \\
& p=v^{T} D f(x)^{T} .
\end{aligned}
$$

In the case of a function $f: M \rightarrow \mathbb{R}, T_{f(x)}^{*} \mathbb{R} \simeq \mathbb{R}$ and the prolongation can be identified with the differential of the map $d f: T M \rightarrow \mathbb{R}$

$$
\hat{f}\left(\xi_{x}\right)=d f_{x}\left(\xi_{x}\right) .
$$

4.3. Variational equations of Lagrangian systems. We now consider the case of $M=T Q$ for some configuration space $Q$, and $L: T Q \rightarrow \mathbb{R}$ a non degenerate Lagrangian for the vector field $Y$ on $T Q$. The prolongation is then the a map $\dot{L}: T(T Q) \rightarrow \mathbb{R}$. As was mentioned at the beginning of this section, the variational vector field $T(Y)$ cannot be viewed as the Lagrangian vector field for the prolongation $\dot{L}$ in an obvious way since the pullback of the canonical 1-form in $T^{*}(T Q)$ to $T(T Q)$ by the Legendre transform $\mathcal{L}(\dot{L})$ of the prolongation $\dot{L}$ is an exact differential.

Instead we consider the D'Alambert phase space consisting of pairs $(q, \epsilon)$ where $q$ is a given configuration and $\epsilon$ is a "virtual displacement". More formally we consider a subbundle , $D \subseteq Q$ then $T D$ an be embeded in $T(T Q)$ under the map $(q, \epsilon, \dot{q}, \dot{\epsilon}) \stackrel{\alpha}{\rightarrow}(q, \dot{q}, \epsilon, \dot{\epsilon})$. Now view the prolongation as a map $\gamma: T D \rightarrow \mathbb{R}$, that is 


$$
\gamma(q, \epsilon, \dot{q}, \dot{\epsilon})=\frac{\partial L}{\partial q}(q, \dot{q}) \epsilon+\frac{\partial L}{\partial \dot{q}}(q, \dot{q}) \dot{\epsilon}
$$

(in fact $T D$ is the natural domain of definition of the prolongation, since the "virtual displacements" are defined by choosing the subbundle).

Notice that the embeding $\alpha$ is well defined since the $\epsilon$ 's are elements of the tangent space $T_{q} Q$, and that $D$ is not necessarily a tangent bundle, that is, it is not necessarily an integrable distribution. Thus we can consider even holonomic or nonholonomic constraints in the specification of $D$.

Theorem 1. Let $\omega_{\gamma}=\mathcal{L}(\dot{L})^{*} \omega_{0}$ be the pullback of the canonical symplectic form on $T^{*} D$ under the Legendre transformation of the prolongation of $L: T Q \rightarrow \mathbb{R}$, viewed as a map $\gamma: T D \rightarrow \mathbb{R}$. Then $T\left(Y_{L}\right)$ viewed as a vector field on $T D$ is a Lagrangian vector field for the energy function $h: T D \rightarrow \mathbb{R}$. In local coordinates $(q, \epsilon, \dot{q}, \dot{\epsilon})$ for $T D$,

$$
h(q, \epsilon, \dot{q}, \dot{\epsilon})=\frac{\partial \gamma}{\partial \dot{q}} \dot{q}-\frac{\partial \gamma}{\partial \epsilon} \epsilon
$$

which coincides with (10).

Proof. We carry on the proof in local coordinates. Let $(q, \epsilon, \dot{q}, \dot{\epsilon})$ be coordinates in $T D$ and $(q, \epsilon, \pi, p)$ coordinates in $T^{*} D$. The canonical 1 -form in $T^{*} D$ is given by $\theta_{0}=\pi d q+p d \epsilon$. The Legendre transform $\mathcal{L}(\gamma)$ is given by

$$
\begin{aligned}
& \pi=\frac{\partial \gamma}{\partial \dot{q}}, \\
& p=\frac{\partial \gamma}{\partial \dot{\epsilon}}=\frac{\partial L}{\partial \dot{q}} .
\end{aligned}
$$

then

$$
\theta_{\gamma}=\frac{\partial \gamma}{\partial \dot{q}} d q+\frac{\partial L}{\partial \dot{q}} d \epsilon
$$

An straightforward computation shows that

$$
\begin{aligned}
\omega_{\gamma}= & \frac{\partial^{2} \gamma}{\partial q_{k} \partial \dot{q}_{j}} d q_{k} \wedge d q_{j}+\frac{\partial^{2} \gamma}{\partial \dot{q}_{k} \partial \dot{q}_{j}} d \dot{q}_{k} \wedge d q_{j}+\frac{\partial^{2} \gamma}{\partial \epsilon_{k} \partial \dot{q}_{j}} d \epsilon_{k} \wedge d q_{j} \\
& +\frac{\partial^{2} \gamma}{\partial \dot{\epsilon}_{k} \partial \dot{q}_{j}} d \dot{\epsilon}_{k} \wedge d q_{j}+\frac{\partial^{2} \gamma}{\partial q_{k} \partial \dot{\epsilon}_{j}} d q_{k} \wedge d \epsilon_{j}+\frac{\partial^{2} \gamma}{\partial \dot{q}_{k} \partial \dot{\epsilon}_{j}} d \dot{q}_{k} \wedge d \epsilon_{j} .
\end{aligned}
$$

Consider the second order equation (or spray)

$$
T\left(Y_{L}\right)=\dot{q} \frac{\partial}{\partial q}+\dot{\epsilon} \frac{\partial}{\partial \epsilon}+C \frac{\partial}{\partial \dot{q}}+D \frac{\partial}{\partial \dot{\epsilon}}
$$

then 


$$
\begin{aligned}
\left.T\left(Y_{L}\right)\right\lrcorner \omega_{\gamma} & =\frac{\partial^{2} \gamma}{\partial q_{k} \partial \dot{q}_{j}}\left(\dot{q}_{k} d q_{j}-\dot{q}_{j} d q_{k}\right)-\frac{\partial^{2} \gamma}{\partial \dot{q}_{k} \partial \dot{q}_{j}} \dot{q}_{j} d \dot{q}_{k}-\frac{\partial^{2} \gamma}{\partial \epsilon_{k} \partial \dot{q}_{j}} \dot{q}_{j} d \epsilon_{k}-\frac{\partial^{2} \gamma}{\partial \dot{\epsilon}_{k} \partial \dot{q}_{j}} \dot{q}_{j} d \dot{\epsilon}_{k} \\
& +\frac{\partial^{2} \gamma}{\partial q_{k} \partial \dot{\epsilon}_{j}} \dot{q}_{k} d \epsilon_{j}+\frac{\partial^{2} \gamma}{\partial \epsilon_{k} \partial \dot{q}_{j}} \dot{\epsilon}_{k} d q_{j}-\frac{\partial^{2} \gamma}{\partial q_{k} \partial \dot{\epsilon}_{j}} \dot{\epsilon}_{j} d q_{k}-\frac{\partial^{2} \gamma}{\partial \dot{q}_{k} \partial \dot{\epsilon}_{j}} \dot{\epsilon}_{j} d \dot{q}_{k} \\
& +\frac{\partial^{2} \gamma}{\partial \dot{q}_{k} \partial \dot{q}_{j}} C_{k} d q_{j}+\frac{\partial^{2} \gamma}{\partial \dot{q}_{k} \partial \dot{\epsilon}_{j}} C_{k} d \epsilon_{j}+\frac{\partial^{2} \gamma}{\partial \dot{\epsilon}_{k} \partial \dot{q}_{j}} D_{k} d q_{j} .
\end{aligned}
$$

The differential of $h$ is

$$
\begin{aligned}
d h & =\left(\frac{\partial^{2} \gamma}{\partial q_{k} \partial \dot{q}_{j}} d q_{k}+\frac{\partial^{2} \gamma}{\partial \dot{q}_{k} \partial \dot{q}_{j}} d \dot{q}_{k}+\frac{\partial^{2} \gamma}{\partial \epsilon_{k} \partial \dot{q}_{j}} d \epsilon_{k}+\frac{\partial^{2} \gamma}{\partial \dot{\epsilon}_{k} \partial \dot{q}_{j}} d \dot{\epsilon}_{k}\right) \dot{q}_{j} \\
& +\frac{\partial \gamma}{\partial \dot{q}_{j}} d \dot{q}_{j}-\left(\frac{\partial^{2} \gamma}{\partial q_{k} \partial \epsilon_{j}} d q_{k}+\frac{\partial^{2} \gamma}{\partial \dot{q}_{k} \partial \epsilon_{j}} d \dot{q}_{k}\right) \epsilon_{j}-\frac{\partial \gamma}{\partial \epsilon_{j}} d \epsilon_{j} .
\end{aligned}
$$

Equating coefficients of $d \epsilon_{k}$ in the equation $T\left(Y_{L}\right)\left\llcorner\omega_{\gamma}=-d h\right.$ one gets

$$
\frac{\partial}{\partial q_{j}}\left(\frac{\partial L}{\partial \dot{q}_{k}}\right) \dot{q}_{j}+\frac{\partial}{\partial \dot{q}_{j}}\left(\frac{\partial L}{\partial \dot{q}_{k}}\right) C_{j}=\frac{\partial L}{\partial q_{k}}
$$

by using the fact that $C_{k}=\ddot{q}_{k}$ (here the dots means derivative with respect to time) wer recover the orignal Lagrange equations

$$
\frac{d}{d t}\left(\frac{\partial L}{\partial \dot{q}_{k}}\right)=\frac{\partial L}{\partial q_{k}} .
$$

Equating coefficients of $d \dot{\epsilon}_{k}$ and $d \dot{q}_{k}$, sendous identities are obtained. Finally equating the coefficients of $d q_{k}$ lead to the linearized equations (7).

\section{EXAMPLES}

A few examples will be presented where the above formulation can be applied.

Geodesic flow. Here the Lagrangian is just the square of the length

$$
L=\frac{1}{2} g_{a b} \dot{q}_{a} \dot{q}_{b}
$$

where $g_{a b}$ is the first fundamental form of the metric. The linearized equations are properly known as Jacobi equations or as equations of geodesic displacement. The prolonged Lagrangian is

$$
\gamma=\frac{\partial g_{a b}}{\partial q_{k}} \epsilon_{k} \dot{q}_{a} \dot{q}_{b}+g_{a b} \dot{q}_{a} \dot{\epsilon}_{b}
$$

The Lagrange equations give the geodesic equations

$$
\ddot{q}_{a}+\Gamma_{a b c} \dot{q}_{b} \dot{q}_{c}=0,
$$

and the equations of geodesic deviation 


$$
\ddot{\epsilon}_{a}=R_{a b c d} \dot{q}_{b} \dot{q}_{d} \epsilon_{c}
$$

where, $\Gamma_{a b c}$ is the affine connection or Christoffel's symbol, $R_{a b c d}$ is the Riemann tensor, and the dots stand in this case for derivatives respect the arc length $s[2][25]$.

Newton's equations. Here

$$
L=\frac{1}{2} m_{a b} \dot{q}_{a} \dot{q}_{b}-V(q),
$$

where $m_{a b}$ is a constant symmetric matrix. The prolonged Lagrangian is

$$
\gamma=-\frac{\partial V}{\partial q_{a}} \epsilon_{a}+m_{a b} \dot{q}_{a} \dot{\epsilon}_{b}
$$

The Lagrange equation

$$
\frac{d}{d t}\left(\frac{\partial \gamma}{\partial \dot{\epsilon}_{k}}\right)=\frac{\partial \gamma}{\partial \epsilon_{k}}
$$

leads to

$$
m_{a k} \ddot{q}_{k}=-\frac{\partial V}{\partial q_{k}},
$$

which are Newton's equations. In the case of the solution being an equilibrium point with the configuration a minimum of the potential, these are the equations of small oscillations. Lagrange equation

$$
\frac{d}{d t}\left(\frac{\partial \gamma}{\partial \dot{q}_{k}}\right)=\frac{\partial \gamma}{\partial q_{k}}
$$

then becomes

$$
m_{a k} \ddot{\epsilon}_{k}+K_{a k} \epsilon_{k}=0,
$$

where

$$
K_{a b}=\frac{\partial^{2} V}{\partial q_{a} \partial q_{b}} .
$$

Plane motions in an arbitrary 2-potential.

Choose a reference curve and let $s=$ arc length, $z=$ normal distance from the curve, and $\rho=$ radius of curvature; then taking $(s, z)$ as generalized coordinates the Lagrangian is [6, 14, 26]

$$
L=\frac{1}{2}\left[\dot{z}^{2}+\frac{\dot{s}^{2}}{\rho^{2}}(\rho+z)\right]-V(z, s) .
$$

The linearized equations obtained by computing the prolonged Lagrangian yields

$$
\ddot{\epsilon}_{z}+\frac{3}{\rho}\left(\frac{\dot{s}^{2}}{\rho}+\frac{\rho}{3} \frac{\partial^{2} V}{\partial z^{2}}\right) \epsilon_{z}=\frac{2 h}{\rho} .
$$


Here is worth to say that we choose the "virtual displacements" $\epsilon_{s}$ and $\epsilon_{z}$ to be planar ones. The constant of motion $h$ can be expressed as

$$
h=\dot{s} \dot{z}\left(\frac{\dot{s}^{2}}{\rho}+\frac{\partial V}{\partial z}\right) \epsilon_{z}+\frac{\partial V}{\partial s} \epsilon_{s} .
$$

We pinpoint that from equation (61) we can see the that a planar orbit is stable, against energy preserving perturbations $(h=0)$, only when the expression between parenthesis is positive [6, 26].

\section{Rotating Lagrangian systems}

Let $q=R(t) Q, \dot{R} R^{-1}=\Omega$ then the Lagrangian of a mechanical system $L(q, \dot{q})=$ $\frac{1}{2}\langle\dot{q}, \dot{q}\rangle-V(q)$ transforms into

$$
L(Q, \dot{Q})=\frac{1}{2}\langle\dot{Q}, \dot{Q}\rangle+\langle\Omega Q, \dot{Q}\rangle-V_{e f}(Q)
$$

in a rotating frame, where $\Omega$ is the rotation matrix and where the effective potential is

$$
V_{e f}(Q)=V(Q)+\frac{1}{2}\langle\mathcal{I} Q, Q\rangle, \quad \mathcal{I} \equiv \text { inertia matrix; }
$$

and $\langle$,$\rangle is the usual inner product in \mathbb{R}^{n}$. The prolonged Lagrangian yields the right linearized equations:

$$
\begin{gathered}
\ddot{Q}+2 \Omega \dot{Q}+\nabla V_{e f}(Q)=0 \\
\ddot{\epsilon}+2 \Omega \dot{\epsilon}+B \epsilon=0 .
\end{gathered}
$$

where $B=H_{e s s} V_{e f}(Q)$.

\section{ACKNOWLEDGEMENTS.}

This work was partially supported by CONACyT and by PAPIIT-UNAM. ALSB wants to thank C. M. Arizmendi and G. Hentshel for very interesting conversations in Atlanta. The authors acknowledge with thanks the useful remarks of G. Sardanashvily of Moscow State University and the suggestions of E. Piña of UAMIztapalapa. Last but not least, HNNY and ALSB dedicate this work to the memory of their beloved friend M. Mec.

\section{REFERENCES}

[1] V. I. Arnold, Mathematical Methods of Classical Mechanics, Springer, New York, 1978.

[2] H. J. Sussman. Lie Brackets, Real Analicity and Geometric Control. In Differential Geometric Control Theory, R.W. Brockett, R.S. Millman, H.J Sussman, eds. Progress in Mathematics, Birkhäusser (1983).

[3] H. J. Sussman. Symmetries and integrals of motion in optimal control. In Geometry and Nonlinear Control and Differential Inclusions, A. Fryszkowski, B. Jakubczyk, W. Respondek, T. Rzezuchowski eds. Banach Center Publications, 32, Institute of Mathematics of the Polish Academy of Sciences, Warsaw Poland, pp. 379-393, (1995).

[4] L. D. Landau and E. M. Lifshiz, Mechanics, Pergamon, Oxford, 1976.

[5] I. C. Percival, Am. Inst. Phys. Conf. Proc. 57 (1979) 302; reprinted in R. S. Mackay and J. D. Meiss (compilators), Hamiltonian Dynamical Systems, Adam Hilger, Bristol, 1987, p. 367.

[6] H. N. Núñez-Yépez, and A. L. Salas-Brito, Phys. Lett. A 275 (2000) 218-222. 
[7] E. T. Whittaker, A treatise on the Analytical Dynamics of Particles and Rigid Bodies, Cambridge University, Cambridge, (1989), fourth edition.

[8] M. P. Do Carmo, Riemannian Geometry, Birkhäuser, Boston, (1992), Ch. 5.

[9] D. E. Soper, Classical Field Theory, John Wiley, New York, (1976).

[10] B. Straughan, Explosive Instabilities in Mechanics, Springer, Berlin, 1998, Ch. 4.

[11] K. M. Case, Proc. Nat. Acad. Sci. U.S.A. 81 (1984) 5893.

[12] G. Giachetta, L. Mangiarotti and G. Sardanashvily, J. Math. Phys., 40, (1999) 1376; G. Giachetta, L. Mangiarotti and G. Sardanashvily, Differential geometry of time-dependent mechanics, arXiv: dg-ga/9702020 (Sections 4.7, 5.5); G. Giachetta, L. Mangiarotti and G. Sardanashvily, New Lagrangian and Hamiltonian Methods in Field Theory, World Scientific, Singapore, (1997), Section 4.9; L. Mangiarotti and G. Sardanashvily, Gauge Mechanics, World Scientific, Singapore, (1998), Section 5.11.

[13] B. Casciaro, M. Francaviglia and V. Tapia, The Second Variation and Jacobi Equations for Second-Order Lagrangians, preprint IC/95/37, International Centre for Theoretical Physics, Trieste, Italia, (1995); B. Casciaro, M. Francaviglia and V. Tapia, Jacobi Equations as Lagrange Equations of the Deformed Lagrangian, preprint IC/95/38, International Centre for Theoretical Physics, Trieste, Italia, (1995); B. Casciaro and M. Francaviglia, Nonlinear Anal. 30, 597 (1997).

[14] A. L. Salas-Brito, Am. J. Phys. 52 (1984) 1012.

[15] P. E. Crouch and A.J. van der Schaft. Variational and Hamiltonian Control Systems. Lecture Notes in Control and Information Sci. 101. Springer-Verlag (1987).

[16] H. Nijmeijer and A. J. van der Schaft. Nonlinear Dynamics Control Systems. Springer (1990).

[17] E. C. G. Sudarshan and N. Mukunda, Classical Dynamics: A Modern Perspective, John Wiley, New York, (1974).

[18] E. Noether, Nachr. Akad. Wiss. Götingen, II, Math.-Physik. Kl., (1918) 235.

[19] K. M. Case, Phys. Rev. Lett. 55, (1985) 445.

[20] C. Nash, Relativistic Quantum Fields, Academic Press, New York (1978).

[21] F. Riesz and B. Sz.-Nagy, Functional Analysis, Dover, New York, (1990).

[22] E. Piña, Contactos, 4 (1991) 57, in Spanish.

[23] J. E. Marsden. Lectures on Mechanics. London Math. Soc. Lect. Notes Ser. 174. Cambridge University Press (1992).

[24] R. Abraham and J. E. Marsden. Foundations of Mechanics. Benjamin/Cummings, New York (1978).

[25] S. Weinberg, Gravitation and Cosmology. Wiley, New York (1972).

[26] S. Chandrasekhar, Principles of Stellar Dynamics, University of Chicago Press, Chicago, (1942) Appendix 2.

(1) Departamento de Física, Universidad Autónoma Metropolitana-Iztapalapa, Apartado Postal 55-534 Iztapalapa 09340 D. F., México.

E-mail address: nyhn@xanum.uam.mx

${ }^{(2)}$ Departamento de Matemáticas, Universidad Autónoma Metropolitana-Iztapalapa, Apartado Postal 55-534, Iztapalapa 09340 D. F., México.

E-mail address: jdf@xanum.uam.mx

(3) Laboratorio de Sistemas Dinámicos, Departamento de Ciencias Básicas, Universidad Autónoma Metropolitana-Azcapotzalco, Apartado Postal 21-726, Coyoacán 04000 D. F., MÉxico.

E-mail address: asb@correo.azc.uam.mx 